\title{
Spontaneous chiral-spin ordering in spin-orbit coupled honeycomb magnets
}

\author{
Qiang Luo $\odot,{ }^{1, *}$ P. Peter Stavropoulos $\odot,{ }^{1}$ Jacob S. Gordon $\odot,{ }^{1}$ and Hae-Young Kee $\oplus^{1,2, \dagger}$ \\ ${ }^{1}$ Department of Physics, University of Toronto, Toronto, Ontario M5S 1A7, Canada \\ ${ }^{2}$ Canadian Institute for Advanced Research, Toronto, Ontario M5G 1Z8, Canada
}

(Received 28 October 2020; revised 1 April 2021; accepted 5 January 2022; published 31 January 2022)

\begin{abstract}
Frustrated magnets with highly degenerate ground states are at the heart of hunting exotic states of matter. Recent studies in spin-orbit coupled honeycomb magnets have generated immense interest in bond-dependent interactions, appreciating a symmetric off-diagonal $\Gamma$ interaction which exhibits a macroscopic degeneracy in the classical limit. Here, we study a generic spin model and discover a chiral-spin (CS) ordering with spontaneously broken time-reversal symmetry near the dominant $\Gamma$ region. The CS phase is demonstrated to possess a staggered chirality relation in different sublattices, and it exhibits gapless excitations, as revealed by the vanishing energy gap and the finite central charge on cylinders. Although there is a vestige of a tiny peak in the corner of the second Brillouin zone, the magnetic order is likely to vanish as the system size increases. Finally, we also attempt to gain insight into the possible topological signature of the CS phase by calculating the dynamic structure factor and the modular $\mathcal{S}$ matrix.
\end{abstract}

DOI: 10.1103/PhysRevResearch.4.013062

\section{INTRODUCTION}

Understanding of the emergent phenomena in exotic honeycomb magnets with strong spin-orbit coupling has triggered an enduring interest in systems that exhibit a type of distinctive ordering due to frustration [1-5]. This in turn leads to a particularly high degeneracy of the ground state, which may be lifted by delicate effects such as quantum fluctuation or entropic difference [6,7]. The magnetically ordered states are often selected from their classical degenerate manifolds; hence, quantum spin liquids (QSLs) are rare and are confined to several specific cases [8,9]. The Kitaev QSL is a rare example which owns fractionalized excitations of Majorana fermions and gauge fluxes [10]. Although it has massive classical degenerate ground states, a finite region of the QSL is demonstrated to exist in the presence of other interactions $[11,12]$.

Along the realization of the Kitaev model in honeycomb materials like $\alpha-\mathrm{RuCl}_{3}$ [13-19], another bond-dependent $\Gamma$ interaction was identified [11], attracting interest because of the inherently strong frustration [20,21]. Its classical limit is known as a classical spin liquid with a macroscopic groundstate degeneracy [20], which includes the antiferromagnetic (AFM) phase, zigzag phase, $120^{\circ}$ phase, and also large-unitcell (LUC) magnetically ordered states $[11,20]$. However, the

\footnotetext{
*Present address: College of Science, Nanjing University of Aeronautics and Astronautics, Nanjing 211106, China.

†hykee@physics.utoronto.ca

Published by the American Physical Society under the terms of the Creative Commons Attribution 4.0 International license. Further distribution of this work must maintain attribution to the author(s) and the published article's title, journal citation, and DOI.
}

quantum ground state of the AFM $\Gamma$ model is more enigmatic, and a few controversial proposals have been reported, including a zigzag phase [22], a nematic paramagnet [23], and a disordered state dubbed $\Gamma$ spin liquid ( $\Gamma$ SL) [24-26].

On the other hand, the chiral-spin (CS) state with spontaneous time-reversal symmetry (TRS) breaking was initially proposed over 30 years ago [27,28] and has been studied in some geometrically frustrated magnets [29-39]. Within the framework of parton mean-field theory [40], which is an approximate method and may overestimate the regime of a certain phase, possible signatures of the CS phase as revealed by nonzero Chern numbers have been presented [41,42]. Beyond that, it has not yet been reported convincingly on a honeycomb lattice except by applying TRS breaking terms such as a magnetic field $[41,43]$ and a three-spin $\hat{\chi}$ interaction [44,45].

In this paper, we study a generic $J K \Gamma \Gamma^{\prime}$ model including the Heisenberg $(J)$, Kitaev $(K)$, and symmetric off-diagonal $\Gamma$ and $\Gamma^{\prime}$ interactions $[11,46]$, focusing on the AFM $\Gamma$ region. The scalar spin chirality [28] is used to search for a CS state. We start with a brief analysis of the chirality on the $\Gamma$ model at the classical level, followed by a joint exact diagonalization (ED) and density matrix renormalization group (DMRG) study of the extended Hamiltonian [47-49]. Strikingly, we uncover a CS phase where the magnetization tends to vanish after an extrapolation while $\hat{\chi}$ remains finite. In addition, it possesses a twofold ground-state degeneracy as a result of the spontaneous TRS breaking.

\section{CLASSICAL $\Gamma$ MODEL WITH STAGGERED SCALAR CHIRALITY}

To understand the origin of finite scalar chirality, we start from a classical $\Gamma$ model which is known to possess a macroscopic ground-state degeneracy. The spin at site $n$ could be 




FIG. 1. Visualization of the classical ground state of the antiferromagnetic (AFM) $\Gamma$ model by a set of Ising variables $\left\{\eta_{n}\right\}$ on individual hexagons. Here, $\mathbf{X}$ (red), $\mathbf{Y}$ (green), and $\mathbf{Z}$ (blue) represent three different bonds shown in Eq. (8), and sites of $A$ and $B$ sublattices are labeled by odd and even numbers, respectively. The scalar chiralities $\chi_{n}^{A}$ and $\chi_{p}^{B}$ are defined as the shaded cyan and pink triangles, respectively.

parameterized by $\hat{\mathbf{S}}_{n}=\left(\eta_{i} a, \eta_{j} b, \eta_{k} c\right)$, where $(a, b, c)=\left(\left|S_{n}^{x}\right|\right.$, $\left.\left|S_{n}^{y}\right|,\left|S_{n}^{z}\right|\right)$, and $\eta_{p}= \pm 1$ is an Ising variable defined on a hexagon $p$, as shown in Fig. 1 [20]. The $\eta$ variables can be divided into three types situated in interpenetrating triangular sublattices. For an $N$-site cluster, there are $N / 2$ local Ising variables, leading to a $2^{N / 2}$-fold ground-state degeneracy in addition to free choices of $(a, b, c)$ with $\sqrt{a^{2}+b^{2}+c^{2}}=S$ [20]. The AFM and zigzag phases belong to a special family where $a=b=c=S / \sqrt{3}$. Nevertheless, most of the configurations are still virgin lands with luxuriant noncoplanar phases.

To study the possible CS ordering out of the degenerate manifold, we introduce the scalar chirality [28]

$$
\hat{\chi}_{i j k}^{\Delta}=\hat{\mathbf{S}}_{i} \cdot\left(\hat{\mathbf{S}}_{j} \times \hat{\mathbf{S}}_{k}\right),
$$

where sites $(i, j, k)$ form an equilateral triangle $(\triangle)$, which belongs to either the $A$ or $B$ sublattice, in the clockwise direction. Depending on the center of triangle, we distinguish two kinds of $\hat{\chi}$ for each sublattice. If it is centered at a site (site 3 , say), we call it $\chi_{p}$; otherwise, it is inside a hexagon and thus termed $\chi_{n}$, see Fig. 1 . For each hexagon, $\chi_{n}$ exhibits an intrinsic staggered relation between $A$ and $B$ sublattices (see the Supplemental Material (SM) [50] for details). Considering the hexagon with $\eta_{7}$ in the center, it is straightforward to check that the scalar chirality for spins at $(9,17,15)$ of the $A$ sublattice is

$$
\begin{aligned}
\hat{\chi}_{9,17,15}^{A}= & \eta_{7}^{3} a^{3}+\eta_{2} \eta_{8} \eta_{11} b^{3}+\eta_{3} \eta_{6} \eta_{12} c^{3} \\
& -a b c \eta_{7}\left(\eta_{3} \eta_{11}+\eta_{2} \eta_{12}+\eta_{6} \eta_{8}\right),
\end{aligned}
$$

and the scalar chirality for spins at $(16,10,8)$ of the $B$ sublat- tice is

$$
\begin{aligned}
\hat{\chi}_{16,10,8}^{B}= & a b c \eta_{7}\left(\eta_{3} \eta_{11}+\eta_{2} \eta_{12}+\eta_{6} \eta_{8}\right) \\
& -\left(\eta_{7}^{3} a^{3}+\eta_{2} \eta_{8} \eta_{11} b^{3}+\eta_{3} \eta_{6} \eta_{12} c^{3}\right),
\end{aligned}
$$

which lead to the intrinsic staggered relation

$$
\hat{\chi}_{9,17,15}^{A}=-\hat{\chi}_{16,10,8}^{B} .
$$

When CS ordering occurs, i.e., $\hat{\chi}_{i j k}^{\Delta}=\hat{\chi}_{l m n}^{\Delta}(\Delta=A, B)$, we find that Ising variables in each triangular sublattice should be equal, leaving only three flavors of $\eta$ s called $\eta_{a}$ (red), $\eta_{b}$ (green), and $\eta_{c}$ (blue). Under such a restriction, $\chi_{p}$ also obeys a staggered relation between $A$ and $B$ sublattices. Furthermore, within each sublattice $\chi_{p}$ and $\chi_{n}$ have the opposite sign, namely, $\chi_{p}^{\Delta}=-\chi_{n}^{\Delta}$. While the $(a, b, c)$ degrees of freedom are still left, they are lifted via an order-by-disorder effect, generating a magnetically ordered state with broken translational symmetry.

To affirm that the magnetic order parameter of the classical CS ordering is finite, it is natural to introduce the static structure factor $(\mathrm{SSF}) \mathbb{S}_{N}(\mathbf{Q})=\sum_{\alpha \beta} \delta_{\alpha \beta} \mathbb{S}_{N}^{\alpha \beta}(\mathbf{Q})$, where

$$
\mathbb{S}_{N}^{\alpha \beta}(\mathbf{Q})=\frac{1}{N} \sum_{i j}\left\langle S_{i}^{\alpha} S_{j}^{\beta}\right\rangle \exp \left[i \mathbf{Q} \cdot\left(\boldsymbol{R}_{i}-\boldsymbol{R}_{j}\right)\right] .
$$

The order parameter is defined as $M_{N}(\mathbf{Q})=\sqrt{\mathbb{S}_{N}(\mathbf{Q}) / N}$, with $\mathbf{Q}$ being the ordering wave vector. In the classical CS phase, its SSF could only peak at the $\mathbf{K}$ point (corner of the first Brillouin zone) and the $\boldsymbol{\Gamma}^{\prime}$ point (corner of the second Brillouin zone) in the reciprocal space, as a consequence of the three-sublattice restriction of $\eta$ variables. In the thermodynamic limit, the magnetic order parameter is defined as $M(\mathbf{Q})=\lim _{N \rightarrow \infty} M_{N}(\mathbf{Q})$, with $\mathbf{Q}=\mathbf{K}$ or $\mathbf{Q}=\boldsymbol{\Gamma}^{\prime}$. After a straightforward calculation, it is found that

$$
\begin{aligned}
M^{2}\left(\boldsymbol{\Gamma}^{\prime}\right) & =\frac{1}{4}\left[\left(a^{2}+b^{2}+c^{2}\right)+2\left(\eta_{a} \eta_{b} a b+\eta_{b} \eta_{c} b c+\eta_{c} \eta_{a} c a\right)\right] \\
& =\frac{1}{4}\left[\left(\bar{a}^{2}+\bar{b}^{2}+\bar{c}^{2}\right)+2(\bar{a} \bar{b}+\bar{b} \bar{c}+\bar{c} \bar{a})\right]
\end{aligned}
$$

and

$$
\begin{aligned}
M^{2}(\mathbf{K}) & =\frac{1}{6}\left[\left(a^{2}+b^{2}+c^{2}\right)-\left(\eta_{a} \eta_{b} a b+\eta_{b} \eta_{c} b c+\eta_{c} \eta_{a} c a\right)\right] \\
& =\frac{1}{6}\left[\left(\bar{a}^{2}+\bar{b}^{2}+\bar{c}^{2}\right)-(\bar{a} \bar{b}+\bar{b} \bar{c}+\bar{c} \bar{a})\right] .
\end{aligned}
$$

Here, $\bar{a}=\eta_{a} a, \bar{b}=\eta_{b} b$, and $\bar{c}=\eta_{c} c$. Mathematically, for any $\bar{a}, \bar{b}, \bar{c} \in[-S, S]$ and $\bar{a}^{2}+\bar{b}^{2}+\bar{c}^{2}=S^{2}$, one can prove that $-S^{2} / 2 \leqslant \bar{a} \bar{b}+\bar{b} \bar{c}+\bar{c} \bar{a} \leqslant S^{2}$. It is worth noting that equality on the left-hand side occurs when $\bar{a}+\bar{b}+\bar{c}=0$, while equality on the right-hand side holds if and only if $\bar{a}=\bar{b}=$ $\bar{c}= \pm S / \sqrt{3}$. Taken together, it is easy to check that $M\left(\Gamma^{\prime}\right) \in$ $[0, \sqrt{3} S / 2]$ and $M(\mathbf{K}) \in[0, S / 2]$, and they cannot be zero simultaneously. Hence, the finite magnetization of the classical CS phase implies that it is a magnetically ordered state.

As we delve into the phase region away from the pure $\Gamma$ limit, we encounter two remarkable sources of quantum fluctuations stemming from huge ground-state degeneracy and competing interactions. While the inherent staggered relations for $\chi_{p}$ and $\chi_{n}$ within each sublattice should still hold, other 

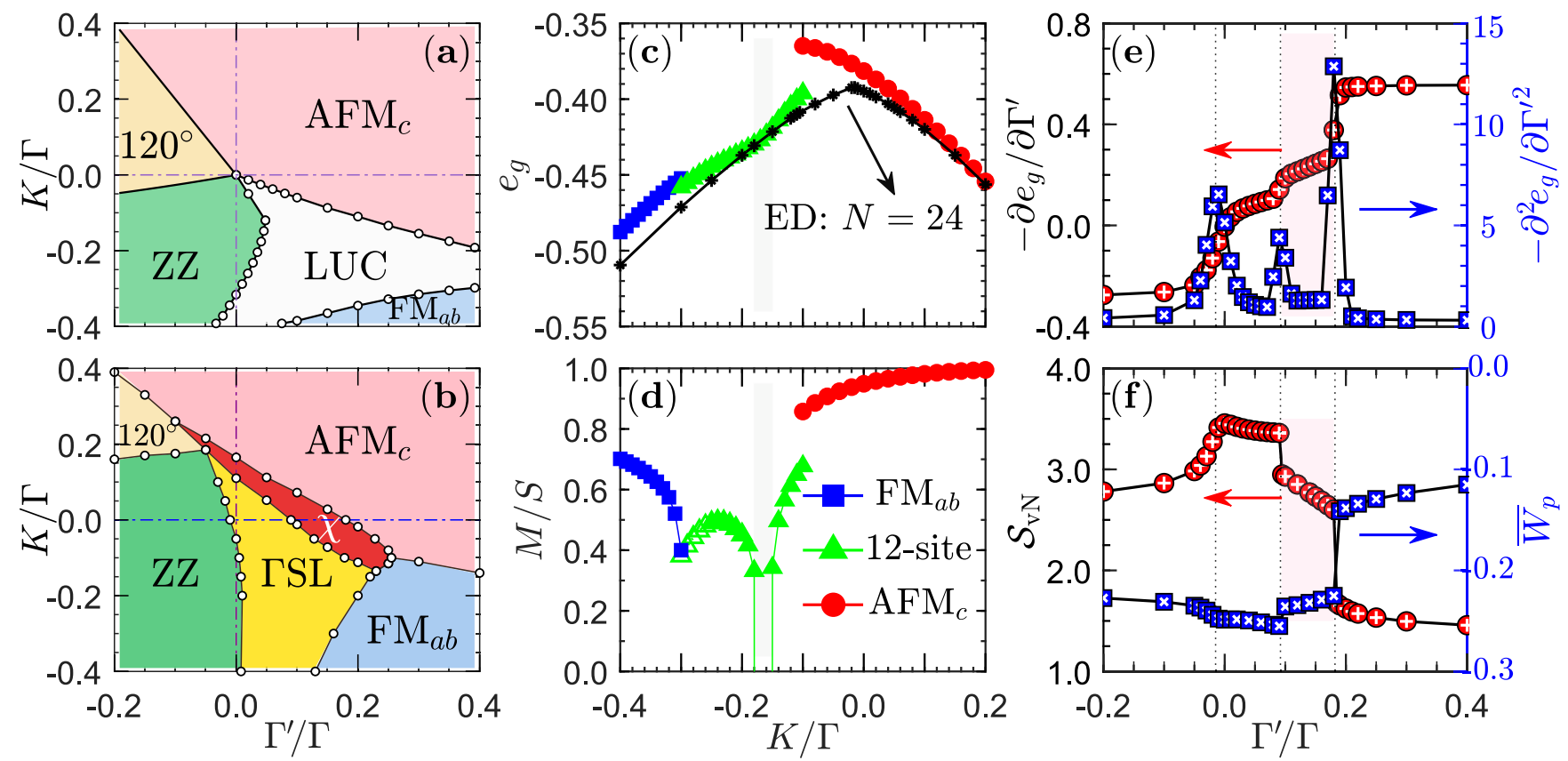

FIG. 2. The (a) classical and (b) quantum phase diagrams of the model in Eq. (8) in the neighborhood of the $\Gamma$ limit. The white region in (a) contains many large-unit-cell (LUC) phases and incommensurate phases near phase boundaries. Details are shown in the Supplemental Material (SM) [50]. In (b), there is a wide region of $\Gamma$ spin liquid ( $\Gamma S L)$ and a chiral-spin (CS) phase marked by $\chi$. (c) and (d) The spin-wave energy $e_{g}$ and magnetization $M$ of the $\mathrm{FM}_{a b}$ phase (blue square), 12-site phase (green triangle), and $\mathrm{AFM}_{c}$ phase (red circle), along the line of $\Gamma^{\prime} / \Gamma=0.2$. In (c), the exact diagonalization (ED) energy on a hexagonal cluster of $N=24$ is shown for comparison (black asterisk). In (d), the open symbol (green triangle) represents the extrapolated magnetization where linear spin-wave theory (LSWT) fails. The gray shadow marks the region where the energy $e_{g}$ is extremely close to the quantum result (black asterisk) and the magnetization $M$ diverges. (e) and (f) Focus on the $\Gamma-\Gamma^{\prime}$ limit in the window of $-0.2 \leqslant \Gamma^{\prime} / \Gamma \leqslant 0.4$. (e) Behaviors of the first-order (red circle) and second-order (blue square) ground-state energy derivatives. (f) Behaviors of the von Neumann entropy $\mathcal{S}_{\mathrm{vN}}$ (red circle) and fluxlike density $\bar{W}_{p}$ (blue square).

constraints could be eased because of strong quantum fluctuations, evoking another quantum CS ordered phase without magnetic ordering. With this in mind, we proceed with ED + DMRG computations to look for numerical evidence.

\section{MODEL HAMILTONIAN AND PHASE DIAGRAMS}

\section{A. Model and methods}

We demonstrate our idea with a paradigmatic spin$\frac{1}{2} J K \Gamma \Gamma^{\prime}$ model whose Hamiltonian reads $[11,46]$

$$
\begin{aligned}
\mathcal{H}= & \sum_{\langle i j\rangle \| \gamma}\left[J \hat{\mathbf{S}}_{i} \cdot \hat{\mathbf{S}}_{j}+K S_{i}^{\gamma} S_{j}^{\gamma}+\Gamma\left(S_{i}^{\alpha} S_{j}^{\beta}+S_{i}^{\beta} S_{j}^{\alpha}\right)\right] \\
& +\Gamma^{\prime} \sum_{\langle i j\rangle \| \gamma}\left[\left(S_{i}^{\alpha}+S_{i}^{\beta}\right) S_{j}^{\gamma}+S_{i}^{\gamma}\left(S_{j}^{\alpha}+S_{j}^{\beta}\right)\right],
\end{aligned}
$$

where $S_{i}^{\gamma}(\gamma=x, y, z)$ is the $\gamma$ component of the spin- $\frac{1}{2}$ operator at site $i$. On $\mathbf{Z}$ bonds $(\alpha, \beta, \gamma)=(x, y, z)$, with cyclic permutation for $\mathbf{X}$ and $\mathbf{Y}$ bonds. The model in Eq. (8) is studied in the vicinity of the $\Gamma$ limit, where $\Gamma=1$ is set as the energy unit. For simplicity, we consider diagonal interactions $J$ and $K$ on equal footing, i.e., $J=K$. The classical phase diagram is obtained by the Luttinger-Tisza analysis [51] and classical Monte Carlo method [52,53] (see Sec. I in the SM [50] for details). The linear spin-wave theory (LSWT) analysis is also utilized when necessary. Depending on the circumstances, three kinds of geometries are employed in
DMRG calculations [47-49]. Firstly, we focus primarily on a $C_{3}$-symmetric hexagonal cluster of $N=24$ with periodic boundary conditions, illustrated in Fig. 1. During the calculation, we keep as many as $m=3000$ block states and execute up to 12 sweeps until the largest truncation error is $<10^{-6}$. Secondly, we also adopt the $Y \mathrm{C} n$ cylinder of $L_{x} \times L_{y}$, which is open along the $L_{x}$ direction and periodic along the $L_{y}$ direction. The letter $Y$ means that one of the bonds is along the perpendicular $\left(L_{y}\right)$ direction, while the letter $n$ represents the number of sites along this direction. Finally, we also consider hexagonal cylinders which are open and periodic in the $\mathbf{e}_{1}(\sqrt{3}, 0)$ and $\mathbf{e}_{2}\left(\frac{\sqrt{3}}{2}, \frac{3}{2}\right)$ directions, respectively. As each unit cell contains two sites, the total number of sites is $N=2 L_{x} L_{y}$, where $L_{x}\left(L_{y}\right)$ represents the number of unit cells along the $\mathbf{e}_{1}$ $\left(\mathbf{e}_{2}\right)$ direction [see inset of Fig. 8(b)].

\section{B. Classical phase diagram}

We begin by mapping out the classical phase diagram of the model in Eq. (8) in the vicinity of the $\Gamma$ limit. As shown in Fig. 2(a), apart from a $\mathrm{FM}_{a b}$ phase (blue) in the right-bottom corner, three magnetically ordered states, the $\mathrm{AFM}_{c}$ phase (pink), the zigzag phase (green), and the $120^{\circ}$ phase (yellow), are selected from the degenerate ground state of the classical $\Gamma$ model [20]. The subscripts imply that the classical moment direction of the $\mathrm{FM}_{a b}$ phase lies in the honeycomb plane, while it is along the out-of-plane direction for the $\mathrm{AFM}_{c}$ phase. In addition, the phase diagram also contains dozens of 
LUC phases and a few incommensurate phases (shown in the white region). The classical energy of the conventional magnetically ordered states (i.e., $\mathrm{AFM}_{c}, 120^{\circ}$, zigzag, and $\mathrm{FM}_{a b}$ ) could be given analytically by the Luttinger-Tisza method. This method is powerful for the determination of classical magnetic ground states, and its application to the spin-orbit coupled model has been outlined in a previous work [51]. Within the framework of the Luttinger-Tisza analysis, we find that the classical energy per site of the $\mathrm{AFM}_{c}$ phase is

$$
\varepsilon_{\mathrm{cl}}^{A F M_{c}}=-\left(\Gamma+2 \Gamma^{\prime}+2 K\right),
$$

and the energy of the spiral $120^{\circ}$ phase is

$$
\varepsilon_{\mathrm{cl}}^{120^{\circ}}=-\frac{1}{2}\left[2\left(\Gamma-\Gamma^{\prime}\right)+K\right] .
$$

The $120^{\circ}$ phase is not favored by the ferromagnetic (FM) Kitaev interaction, which then gives way to the zigzag phase whose energy is

$$
\varepsilon_{\mathrm{cl}}^{\text {zigzag }}=-\frac{1}{4}\left(\mathcal{R}+\sqrt{8 \Gamma^{2}+\mathcal{R}^{2}}\right),
$$

where $\mathcal{R}=\Gamma-2 \Gamma^{\prime}-2 K$. The classical moment direction of the zigzag phase is tilted away from the $a b$ plane in the crystalline reference frame, and the tilted angle is known to depend on the interaction parameters [51].

As can be seen from Fig. 2(a), in the left part of the phase diagram, where $\Gamma^{\prime}<0$, the $120^{\circ}$ phase is sandwiched between the $\mathrm{AFM}_{c}$ phase and the zigzag phase. For the $\mathrm{AFM}_{c^{-}}$ $120^{\circ}$ transition, the transition boundary is

$$
K_{c, u}=-2 \Gamma^{\prime},
$$

while for the zigzag- $120^{\circ}$ transition, it is

$$
K_{c, l}=\frac{\sqrt{49 \Gamma^{2}-60 \Gamma \Gamma^{\prime}+36 \Gamma^{\prime 2}}-\left(7 \Gamma-6 \Gamma^{\prime}\right)}{6} .
$$

Specifically, when $\left|\Gamma^{\prime} / \Gamma\right| \ll 1$, Eq. (13) is reduced to

$$
K_{c, l}=\frac{2}{7}\left[\Gamma^{\prime}+\left(\frac{6}{7} \Gamma^{\prime}\right)^{2}\right]+\mathcal{O}\left(\Gamma^{\prime 3}\right) .
$$

On the other side, where $\Gamma^{\prime}>0$, the $\mathrm{AFM}_{c}$ phase occupies the majority region of the phase diagram. However, for a large enough FM Kitaev interaction, there is a $\mathrm{FM}_{a b}$ phase whose energy is

$$
\varepsilon_{\mathrm{cl}}^{\mathrm{FM}_{a b}}=-\frac{1}{2}\left(\Gamma+2 \Gamma^{\prime}\right)+2 K .
$$

One should notice that there is not a direct transition between the $\mathrm{AFM}_{c}$ phase and the $\mathrm{FM}_{a b}$ phase for modest $\Gamma^{\prime}$. Instead, many magnetically ordered LUC states are demonstrated to exist in between. These LUC phases are unstable against quantum fluctuation, providing a fertile playground for the realization of disordered phases at the quantum level [54].

\section{Spin-wave analysis in the $L U C$ region}

The simplest way to roughly demonstrate that LUC orderings could be melted by quantum fluctuations is perhaps the LSWT, which is an efficient method that has been applied successfully in various spin-orbit coupled models on the honeycomb lattice $[15,19,55]$. In this method, the spins are rewritten as the bosonic creation and annihilation operators, and the Hamiltonian $\mathcal{H}$ is reduced to a quadratic form $\mathcal{H}_{\mathrm{sw}}$ up to $\mathcal{O}(1 / S)$. The spin-wave dispersion $\omega_{\mathbf{q} v}$ and the corresponding eigenvector $\mathbf{v}_{\mathbf{q}}^{(v)}$ are readily obtained. Here, $\mathbf{q}$ is the wave vector in the reciprocal space, and $v=1,2, \ldots, n_{s}$, with $n_{s}$ being the number of sites in the unit cell. As a result, the spin-wave energy $e_{g}$ is given by

$$
e_{g}=S(S+1) \varepsilon_{\mathrm{cl}}+\frac{S}{2 n_{s}} \sum_{\{v\} \in n_{s}} \int \frac{d^{2} \mathbf{q}}{(2 \pi)^{2}} \omega_{\mathbf{q} v},
$$

and the classical moment $M$ is

$$
\frac{M}{S}=1-\frac{1}{n_{s} S} \sum_{\{v\} \in n_{s}} \int \frac{d^{2} \mathbf{q}}{(2 \pi)^{2}}\left|\mathbf{v}_{-\mathbf{q}}^{*(v)}\right|^{2}
$$

To get started, it is useful to recall the classical Monte Carlo result of the model in Eq. (8). For this purpose, let us focus on the transitions along the line of $\Gamma^{\prime}=0.20$, which is known to host a $\mathrm{FM}_{a b}$ phase when $K<-0.34$ and an $\mathrm{AFM}_{c}$ phase when $K>-0.11$ (for details, see Fig. S2 in the SM [50]). In between, there are three LUC phases whose magnetic unit cells are 44, 12, and 24 sites, respectively, with the increase of $K$. Within the LUC region, the extent of the remaining two is considerably smaller than the middle 12-site phase, and thus, they are ignored tentatively. Further, the classical magnetic moment directions are essential for the spin-wave analysis. For the $\mathrm{AFM}_{c}$ phase, it is simply along the c[111] direction. By contrast, for the $\mathrm{FM}_{a b}$ phase, the spins are free to rotate in the $a b$ plane as a consequence of an emergent $U(1)$ symmetry. However, this continuous $U(1)$ symmetry is fragile against the quantum fluctuation and breaks to a discrete $\mathbb{Z}_{2}$ symmetry with an energetically favored moment direction along the $\mathbf{b}[\overline{1} 10]$ axis. We stress that such an order-by-disorder effect has also been explored in the stripe phase [26,56]. The magnetic moment of the 12-site phase is more complicated, but it can be determined by the Monte Carlo method or the energy optimization method illustrated in the SM [50].

The LSWT calculations of the energy $e_{g}$ and magnetization $M$ on the $\mathrm{FM}_{a b}$ (blue square), 12-site (green triangle), and $\mathrm{AFM}_{c}$ (red circle) phases are shown in Figs. 2(c) and 2(d), respectively. In Fig. 2(c), the ED energy on a hexagonal cluster of $N=24$ is shown for comparison. In the whole parameter region, the spin-wave energy $e_{g}$ is higher than the quantum result (black asterisk), and there is a magnetization reduction in the magnetically ordered phases, which is more pronounced in the 12-site phase. However, the most striking observation is that, in the narrow region of $-0.18 \leqslant K \leqslant-0.15$, the spin-wave energy (green triangle) is extremely close to the ED energy (black asterisk). In addition, we find that the magnetization $M$ diverges as it goes to a (infinite) negative value, indicating a strong quantum fluctuation which melts the magnetic ordering. Notably, this region is consistent with the $\Gamma$ SL phase in the quantum phase diagram, cf. Fig. 2(b). Thus, our LSWT results suggest the the LUC phases are unstable against quantum fluctuations, giving rise to the disordered phases in certain region. 



FIG. 3. Typical contour plots of the static structure factors (SSFs) in the phase diagram shown in Fig. 2(b), which includes (a) the zigzag phase with $\left(K, \Gamma, \Gamma^{\prime}\right)=(0.0,1.0,-0.1)$, (b) the $120^{\circ}$ phase with $\left(K, \Gamma, \Gamma^{\prime}\right)=(0.3,1.0,-0.2)$, (c) the $\mathrm{FM}_{a b}$ phase with $\left(K, \Gamma, \Gamma^{\prime}\right)=(-0.2,1.0,0.3),(\mathrm{d})$ the $\mathrm{AFM}_{c}$ phase with $\left(K, \Gamma, \Gamma^{\prime}\right)=$ $(0.0,1.0,0.3)$, (e) the $\Gamma$ spin liquid $(\Gamma \mathrm{SL})$ with $\left(K, \Gamma, \Gamma^{\prime}\right)=$ $(0.0,1.0,0.05)$, and (f) the chiral-spin $(\mathrm{CS})$ phase with $\left(K, \Gamma, \Gamma^{\prime}\right)=$ $(0.0,1.0,0.15)$.

\section{Quantum phase transitions}

The quantum phase diagram is shown in Fig. 2(b), which contains six distinct phases, and four of them are magnetically ordered as identified in the classical case. The typical SSFs $\mathbb{S}(\mathbf{Q})$ of the four magnetically ordered phases on a 24-site hexagonal cluster are illustrated in Figs. 3(a)-3(d). It is clearly shown that the zigzag phase [panel (a)], the $120^{\circ}$ phase [panel (b)], the $\mathrm{FM}_{a b}$ phase [panel (c)], and the $\mathrm{AFM}_{c}$ phase [panel (d)] display the ordering wave vectors at the $\mathbf{M}, \mathbf{K}, \boldsymbol{\Gamma}$, and $\boldsymbol{\Gamma}^{\prime}$ points in the Brillouin zone, respectively [for the illustration of the high-symmetry points, see inset of Fig. 4(b)].


FIG. 4. Order parameters $M_{N}(\mathbf{Q})$ for the zigzag phase (red square) and $\mathrm{AFM}_{c}$ phase (blue circle) with $\mathbf{Q}=\mathbf{M}$ and $\boldsymbol{\Gamma}^{\prime}$, respectively, in the $\Gamma-\Gamma^{\prime}$ limit. (a) and (b) Results obtained on the 24-site hexagonal cluster and YC6 cylinder of $12 \times 6$, respectively. The inset of (b) shows the Brillouin zone with high-symmetry points.
Throughout the phase diagram, it also acquires two emergent phases which do not have a classical analogy. One is the $\Gamma$ SL that can be compatible with a small positive $\Gamma^{\prime}$ interaction. It is named after the ground state of the pure $\Gamma$ model that is proposed to be a gapless QSL [26]. As shown in Fig. 3(e), there are only soft peaks in the reciprocal space, and these peaks are expected to be more diffusive with the increase of the system size. The other is a CS ordered phase that appears in a narrow slot between the $\Gamma \mathrm{SL}$ and the $\mathrm{AFM}_{c}$ phase. The $\mathbb{S}(\mathbf{Q})$ is diffusive inside the first Brillouin zone, while a tiny peak at the $\Gamma^{\prime}$ point appears in the second Brillouin zone [see Fig. 3(f)]. We note that such a peak is an indication of the TRS breaking.

Below, we enumerate the methods to determine the phase boundaries and identify the nature of phases therein. Without loss of generality, we consider the $\Gamma-\Gamma^{\prime}$ line to focus on the $\Gamma$ SL and CS phase. The basic quantity is the ground-state energy $e_{g}=E_{g} / N$ and its derivatives, which exhibit jump or peak at the transition point, depending on the order of the phase transition. As shown in Fig. 2(e), the phase loci are pinpointed by the first- and second-order derivatives of the ground-state energy. There are three consecutive phase transitions at $\Gamma^{\prime} \approx-0.015,0.095$, and 0.185 , respectively. Further, we can use the von Neumann entanglement entropy $\mathcal{S}_{\mathrm{vN}}$ to distinguish different phases. It is defined as $\mathcal{S}_{\mathrm{vN}}=$ $-\operatorname{tr}\left(\rho_{s} \ln \rho_{s}\right)$, where $\rho_{s}$ is the reduced density matrix obtained by tracing over the degrees of freedom in the other halfsubsystem. Generally, the transition could be revealed by its turning point or pinnacle/jump position. Moreover, we can define a hexagonal plaquette operator $\hat{W}_{p}=2^{6} S_{1}^{x} S_{2}^{y} S_{3}^{z} S_{4}^{x} S_{5}^{y} S_{6}^{z}$ on a plaquette $p$ and then introduce the fluxlike density $\bar{W}_{p}=$ $\sum_{p}\left\langle\hat{W}_{p}\right\rangle / N_{p}$, where $N_{p}=N / 2$ is the number of hexagonal plaquettes. Over the years, $\bar{W}_{p}$ has emerged as a sensitive probe to capture quantum phase transitions in a variety of systems [12,26,57,58]. Figure 2(f) shows the behaviors of the entanglement entropy $\mathcal{S}_{\mathrm{vN}}$ (red circle) and fluxlike density $\bar{W}_{p}$ (blue square), and it is found that they yield the same transition points to that of the energy derivatives. Remarkably, the sharp jumps in $\mathcal{S}_{\mathrm{vN}}$ and $\bar{W}_{p}$ at the left and right boundaries of the CS phase indicate that the transitions therein are of first order.

After having determined the transition points, here, we go into the phases via the SSFs. For $\Gamma^{\prime} \lesssim-0.015$, the SSF $\mathbb{S}_{N}(\mathbf{Q})$ displays distinct peaks at the $\mathbf{M}$ point in the first Brillouin zone, while for $\Gamma^{\prime} \gtrsim 0.185$, dominating peaks located at the $\Gamma^{\prime}$ point in the second Brillouin zone appear. These results advocate the semiclassical analysis that AFM $\Gamma^{\prime}$ interaction favors the $\mathrm{AFM}_{c}$ phase, while even a tiny $\mathrm{FM} \Gamma^{\prime}$ interaction could stabilize the zigzag phase $[55,57]$. The order parameters $M_{N}(\mathbf{Q})$ for the zigzag phase (blue square) and the $\mathrm{AFM}_{c}$ phase (red circle) are shown in Fig. 4(a), and two intermediate phases are found in between. One is recognized as the $\Gamma \mathrm{SL}$ since the SSF is diffusive with soft peaks and it incorporates the ground state of the $\Gamma$ model which is proposed to be a gapless QSL [26]. The other is referred to as the CS ordered phase whose nature will be clarified later.

To underpin the conclusions drawn from the calculations on the 24-site cluster under full periodic boundary conditions, we next present the results on the YC6 cylinder, which strikes 


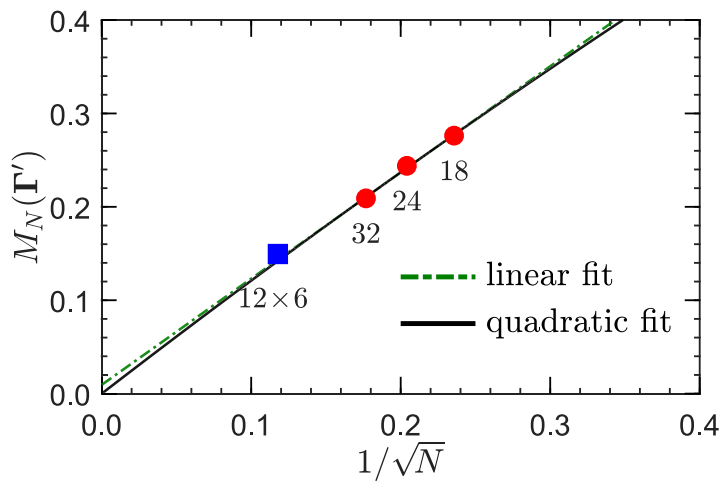

FIG. 5. Extrapolation of $M_{N}\left(\Gamma^{\prime}\right)$ at $\Gamma^{\prime} / \Gamma=0.15$ under hexagonal clusters of $N=18,24$, and 32 (red circle). The blue dash-dot line represents the linear fitting, while the black solid line stands for the quadratic fitting. The magnetic order under a $12 \times 6$ cylinder with $N=72$ (blue square) is plotted for comparison.

a balance between bidimensionality and capacity to converge the DMRG calculation. We note that the $12 \times 6 \mathrm{YC}$ cylinder represents a superior geometry since the number of sites contained is the multiple of $2,4,6,12$, and 18 , which could accommodate many conventional and LUC orderings and thus reduces the artificial effect. The order parameters of the zigzag and $\mathrm{AFM}_{c}$ phases on the $Y \mathrm{C} 6$ cylinder of $12 \times 6$ are shown in Fig. 4(b), from which we can tell that the transition points are almost the same as those of the 24-site case, corroborating the robustness of the two emergent phases.

In the CS ordered phase, the soft peak at the $\boldsymbol{\Gamma}^{\prime}$ point in the reciprocal space is reminiscent of TRS breaking. However, the magnitude of the peak decreases with the growing of the system size. Figure 5 shows the extrapolation of the magnetic order parameter $M_{N}\left(\boldsymbol{\Gamma}^{\prime}\right)$ at $\Gamma^{\prime}=0.15$ on hexagonal clusters of $N=18,24$, and 32 , and the value of the magnetic order parameter on a $12 \times 6$ cylinder is also plotted for comparison. It is found that $M_{N}\left(\boldsymbol{\Gamma}^{\prime}\right)$ shows a roughly linear decrease with $1 / \sqrt{N}$, with an estimated value of $0.01(0.00)$ for the linear (quadratic) fitting when $N \rightarrow \infty$, signifying the absence of long-range magnetic ordering in the ground state. These results suggest that the CS phase is likely a disordered phase, which stands out in remarkable contrast to the classical CS ordering which is characterized by a nonzero magnetization.

\section{THE CS ORDERED STATE}

\section{A. Degeneracy and staggered chirality}

In this section, we adopt the momentum-resolved ED calculation and the large-scale DMRG calculation, in conjunction with the symmetry analysis, to decipher the nature of the CS ordered phase. Without loss of generality, we proceed to focus on the $\Gamma-\Gamma^{\prime}$ line where the CS phase is sandwiched between the $\Gamma \mathrm{SL}$ and the $\mathrm{AFM}_{c}$ phase. Before studying the excitations of the CS phase, it is useful to recall that the $\Gamma$ SL is gapless albeit with a small energy gap retained in finite-sized systems [26], and the $\mathrm{AFM}_{c}$ phase is gapped with a doubly degenerate ground state. Figure 6 shows the first two energy gaps $\Delta_{1,2}=E_{1,2}-E_{g}$ on a 24 -site cluster in the $\Gamma-\Gamma^{\prime}$ model. The intervening CS phase has a twofold degenerate ground

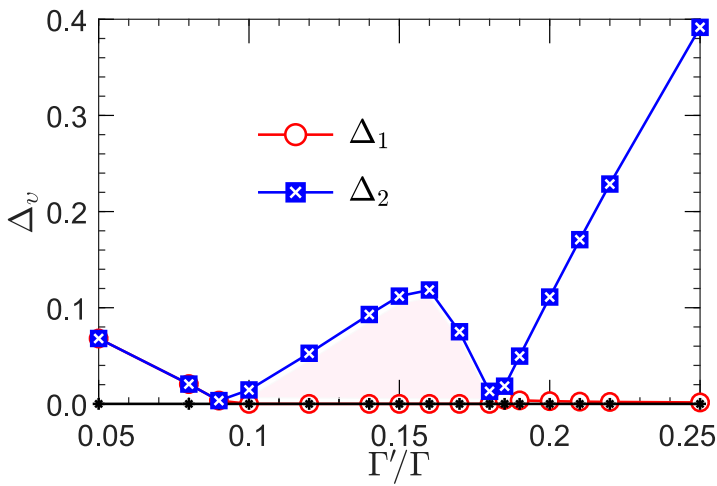

FIG. 6. The first (red circle) and second (blue square) energy gaps $\Delta_{1,2}$ in the range of $0.05 \leqslant \Gamma^{\prime} / \Gamma \leqslant 0.25$. The pink region marks the chiral-spin (CS) phase, which has a finite energy gap in the 24-site cluster.

state, which is well separated from the excited states with a finite energy gap $\Delta_{2}$ due to the small system size. Under the 24-site hexagonal cluster, the doubly degenerate ground state is protected by the combination of TRS $\hat{\mathcal{T}}$, inversion symmetry $\hat{\mathcal{P}}$, and $\hat{C}_{2 b}$ about the crystallographic $\hat{\mathbf{b}}$ direction. To target a purified ground state without breaking TRS abruptly, we add a tiny pinning field $h_{z}=10^{-3}$ with opposing sign at endpoint sites (i.e., sites 1 and 24 shown in Fig. 1) which belong to different sublattices. The energy splitting is approximately of $\mathcal{O}\left(h_{z}^{2}\right)$, which is far less than the corresponding energy gap $\Delta_{2}$ and thus only slightly perturbs the ground state. After locking a specific one, we gradually reduce the strength of $h_{z}$ and do not stop sweeping until $h_{z}$ is vanishingly small.

Apart from the 24-site case, we have also checked the ground-state degeneracy of the CS phase on 18- and 32-site hexagonal clusters. Table I shows the first five energy levels on the three clusters where $\Gamma^{\prime}=0.15$ is taken as an example. Apparently, the ground-state degeneracy is always twofold in these cases. However, the energy gap $\Delta_{2}$ is not monotonously varying with the increase of the system size. The energy gap is $0.012,0.112$, and 0.0002 for hexagonal clusters of $N=18$, 24 , and 32 , respectively, suggesting a likely vanishing energy gap for large enough systems. The existence of the gapless excitations is also borne out by the entanglement entropy scaling that will be shown later.

Next, we turn to unveil the unusual restrictions on the scalar spin chirality in the CS phase. For any purified ground state, the spatial distributions of $\chi_{p}$ (blue square) and $\chi_{n}$ (red circle) on a 24-site cluster at $\Gamma^{\prime}=0.15$ are shown in Fig. 7(a). For either type of chirality, there are sign differences between $A$ and $B$ sublattices, in accordance with Eq. (4). Intriguingly, the signs of $\chi_{p}$ and $\chi_{n}$ are identical within each sublattice, and their magnitudes are unequal. We emphasize that these properties are distinct from the classical CS ordering stemming from the degenerate ground state of the classical $\Gamma$ model, indicating the vital role played by the $\Gamma^{\prime}$ interaction. Pertinently, effects of a small AFM $\Gamma^{\prime}$ term in honeycomb magnets have been studied recently [59,60]. On the other hand, the other degenerate ground state has an opposing sign structure of the scalar chirality, and it is zero for excited states [50]. Figure 7(b) shows the magnitudes of $\chi_{p}$ and $\chi_{n}$ 
TABLE I. The first five energy levels $E_{v}(v=0-4)$ at $\Gamma^{\prime} / \Gamma=0.15$ in the $\Gamma-\Gamma^{\prime}$ model on hexagonal clusters of $N=18,24$, and 32 . The energy gap is defined as $\Delta_{2}=E_{2}-E_{0}$.

\begin{tabular}{lccc}
\hline \hline & $N=18$ & $N=24$ & $N=32$ \\
\hline$E_{0}$ & -6.6497245386 & -9.0381902795 & -12.06667 \\
$E_{1}$ & -6.6497245386 & -9.0381902795 & -12.06667 \\
$E_{2}$ & -6.6376518389 & -8.9261211865 & -12.06655 \\
$E_{3}$ & -6.6099455586 & -8.8509767453 & - \\
$E_{4}$ & -6.5769370525 & -8.8509767453 & - \\
$\Delta_{2}$ & 0.0120726997 & 0.1120690930 & 0.00012 \\
\hline \hline
\end{tabular}

in the region of $\Gamma^{\prime} \in[0.05,0.25]$, highlighting the CS phase characterized by a finite chirality of roughly 0.03 (for $\chi_{p}$ ) or 0.02 (for $\chi_{n}$ ). We also calculate the chiral distribution on hexagonal clusters of $N=18$ and 32 at $\Gamma^{\prime}=0.15$, and we find that the chirality is fairly stable with a negligible finitesized effect. To summarize, our study on the magnetic order parameters and the scalar chirality leads us to the conclusion that the CS phase is a magnetically disordered phase but with a rank-2 chiral ordering. To gain insight into the chirality relations, we denote the ground-state doublet as $\left\{\left|\mathbf{K}_{1}\right\rangle,\left|\mathbf{K}_{2}\right\rangle\right\}$, with $\mathbf{Q}=\mathbf{K}_{1,2}$ being the total momentum of the ground states [see inset of Fig. 4(b)]. Since the composite operator $\hat{\Theta}=\hat{\mathcal{T}} \hat{\mathcal{P}}$ relates $\left|\mathbf{K}_{1,2}\right\rangle$ to itself, we find a staggered relation between inversion-related chiral operators in each ground state:

$$
\left\langle\mathbf{K}_{1,2}\left|\hat{\chi}^{A / B}\right| \mathbf{K}_{1,2}\right\rangle=-\left\langle\mathbf{K}_{1,2}\left|\hat{\chi}^{B / A}\right| \mathbf{K}_{1,2}\right\rangle .
$$

On the other hand, since $\hat{C}_{2 b}$ relates the different ground states, we have a staggered relation between $\hat{C}_{2}$-related chiral operators in opposite ground states:

$$
\left\langle\mathbf{K}_{1}\left|\hat{\chi}^{A / B}\right| \mathbf{K}_{1}\right\rangle=-\left\langle\mathbf{K}_{2}\left|\hat{\chi}^{A / B}\right| \mathbf{K}_{2}\right\rangle .
$$
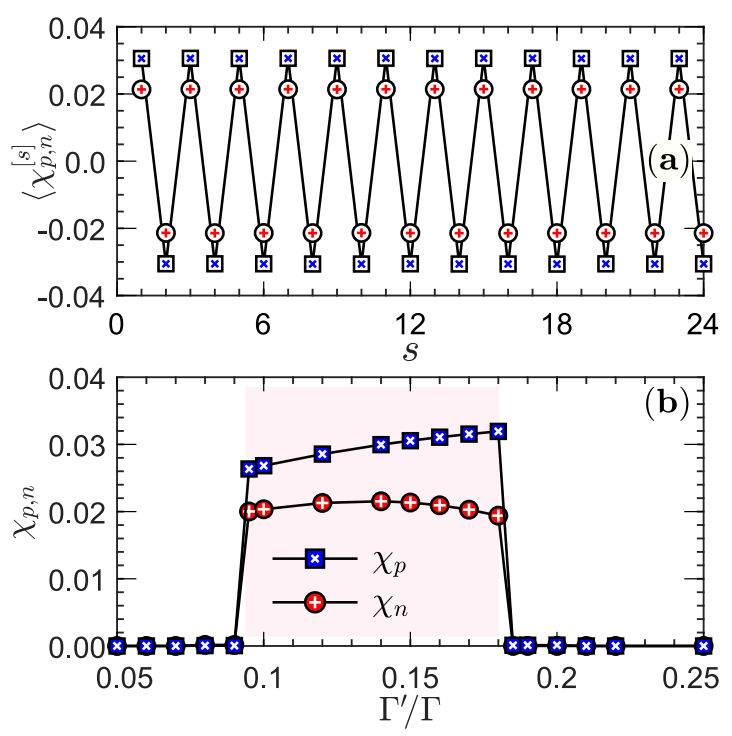

FIG. 7. (a) Chiral distribution of $\chi_{p}^{[s]}$ (blue square) and $\chi_{n}^{\left[s^{\prime}\right]}$ (red circle) at $\Gamma^{\prime} / \Gamma=0.15$ in the $\Gamma-\Gamma^{\prime}$ model. The site number $s$ is shown in Fig. 1, while $s^{\prime}$ denotes $\eta$ with the same $\chi_{n}^{B}-\chi_{n}^{A}$ pattern as $\chi_{p}$. (b) The two kinds of chirality $\chi_{p}$ (blue square) and $\chi_{n}$ (red circle) in the range of $0.05 \leqslant \Gamma^{\prime} / \Gamma \leqslant 0.25$.
Note that none of the symmetries can interchange the $n$ - and $p$ type chiral operators, so generically, their expectation values will differ. Taken together, these constraints account for the observed structures of the chirality in the degenerate subspace.

\section{B. Entropy and central charge}

Previously, we have inferred that the CS phase is gapless based on the behavior of the energy gap with respect to the system size. To further affirm gapless excitations in the CS phase, we now calculate the entanglement entropy $\mathcal{S}_{\mathrm{vN}}$ on the three-leg cylinder with a length of $L_{x}$ along the open direction. Although the other direction is periodic, two distinct connections termed RC3-0 and RC3-1, which correspond to identifying site $x$ with sites 0 and 1 [see the inset of Fig. 8(b)], are treated for the sake of central charge. For a one-dimensional critical system, it is well established that $\mathcal{S}_{\mathrm{vN}}$ obeys the following formula:

$$
\mathcal{S}_{\mathrm{vN}}\left(L_{x}, x\right)=\frac{c}{6} \ln \left[\frac{2 L_{x}}{\pi} \sin \left(\frac{\pi x}{L_{x}}\right)\right]+c^{\prime},
$$

where $x$ is the length of the subsystem $[61,62]$. Here, $c$ is the central charge, and $c^{\prime}$ is a nonuniversal constant. If the length of the subsystem is $\frac{1}{2}$ of the whole system, i.e., $x=L_{x} / 2$, then the entropy exhibits the following behavior:

$$
\mathcal{S}_{\mathrm{vN}}\left(L_{x}, L_{x} / 2\right)=\frac{c}{6} \ln \left(\frac{2 L_{x}}{\pi}\right)+c^{\prime} .
$$

Figure 8(a) shows the entanglement entropy $\mathcal{S}_{\mathrm{vN}}(l)$ as a function of site index $l(l=1,2,3, \ldots, N)$ under the cylinder of $2 \times 36 \times 3$. Since the connection is of RC3-0 type, the gapless excitations could not propagate smoothly along the snakelike path, making the entropy somewhat chaotic. We performed up to 24 sweeps during the DMRG calculation until the entropy did not change in the first few digits. Figure 8(b) shows the entanglement entropy scaling according to Eq. (21) on a series of three-leg cylinders. When the length of the cylinder is small, the boundary effect is significant and the entropy deviates from the scaling law. For a long enough cylinder with $L_{x} \geqslant 24$, the central charge is estimated as 1.2 , which is close to 1 within the numerical precision.

By contrast, in Fig. 8(c), we show the entanglement entropy $\mathcal{S}_{\mathrm{vN}}(l)$ under the cylinder of $2 \times 36 \times 3$ with the connection of RC3-1 type. In this case, the uppermost site $6 x$ at the $x$ column is connected to the lowest site $6 x+1$ at the $(x+1)$ column. Thus, the entanglement of the whole system is enhanced, and the entanglement entropy is higher than that 

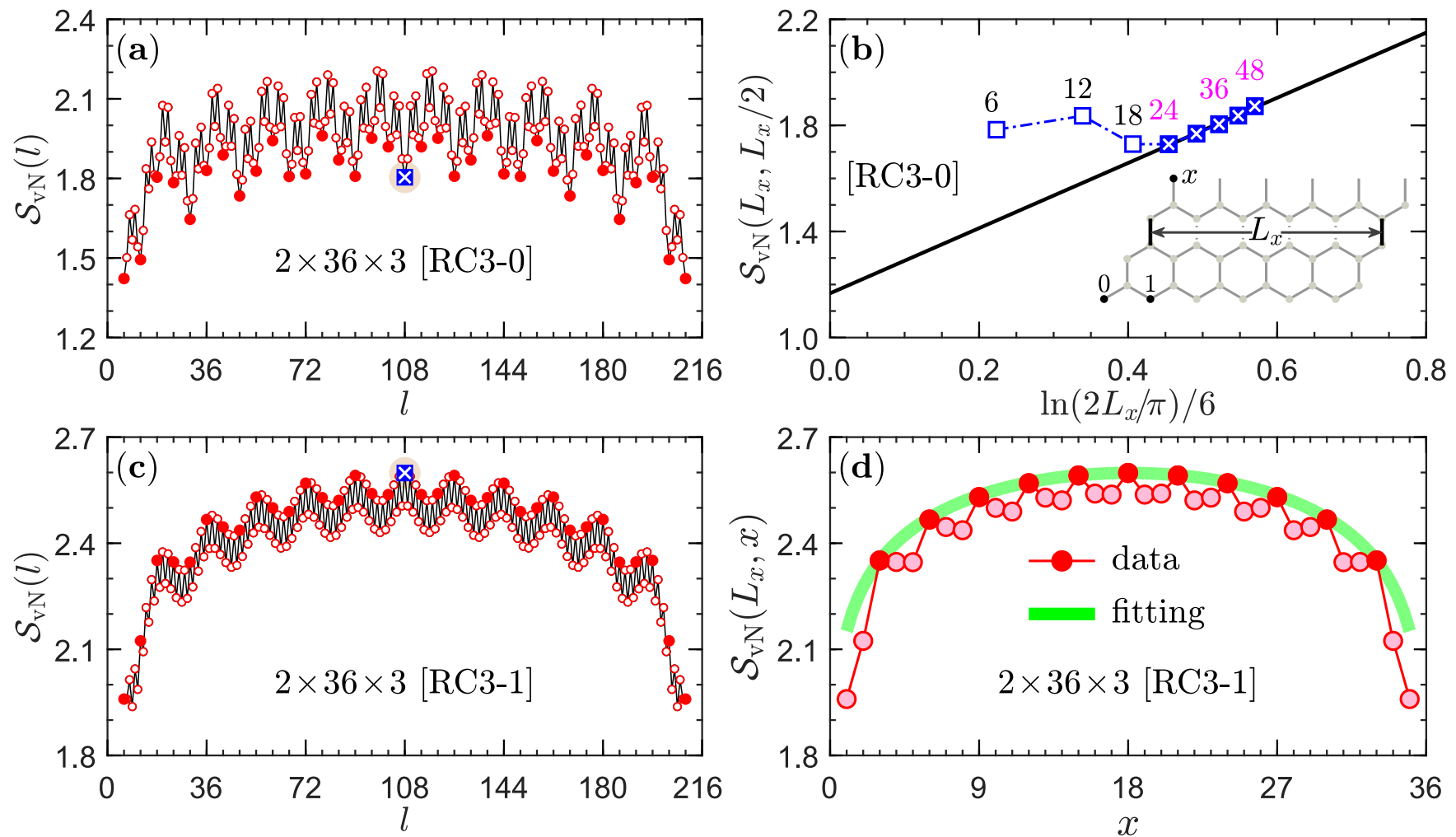

FIG. 8. The entanglement entropy and its scaling on different three-leg cylinders at $\Gamma^{\prime} / \Gamma=0.15$ in the $\Gamma$ - $\Gamma^{\prime}$ model. (a) Entanglement entropy $\mathcal{S}_{\mathrm{vN}}(l)$ of a consecutive segment of length $l$ on a $2 \times 36 \times 3$ cylinder with the connection of RC $3-0$. The solid symbols represent the neat edge-cutting with $l$ being a multiple of 6 (i.e., the number of the sites along each column). The bipartite entanglement entropy with $l=N / 2$ is marked as a blue square. (b) The bipartite entanglement entropy $\mathcal{S}_{\mathrm{vN}}\left(L_{x}, L_{x} / 2\right)$ under a series of $2 \times L_{x} \times 3$ cylinders with the connection of RC3-0. The central charge is fitted as 1.2 based on the results at $L_{x}=24,30,36,42$, and 48 . The inset shows the geometry of the three-leg cylinders. Depending on the way to connect the upper and lower boundaries, the cylinders are called RC3-0 and RC3-1, respectively, when connecting the site $x$ (upper) to sites 0 and 1 (lower). (c) The same as (a) but for the connection of RC3-1. (d) Extracting the central charge $c$ from the entanglement entropy $\mathcal{S}_{\mathrm{vN}}\left(L_{x}, x\right)$ on a $2 \times 36 \times 3$ cylinder with the connection of RC3-1. The fitting formula is Eq. (20), and the central charge is estimated as 1.1 .

of Fig. 8(a). Nevertheless, the advantage of this connection is that the gapless excitations could propagate smoothly from one edge to another, and thus, there are two distinct branches of the entanglement entropy in the $A / B$ sublattices of the honeycomb lattice. In Fig. 8(d), we show the entanglement entropy scaling on the $2 \times 36 \times 3$ cylinder with the RC3-1 connection according to Eq. (20). Our best fitting suggests that the central charge is $\sim 1.1$, which is again very close to 1 . Hence, a finite central charge of 1 on the three-leg cylinder supports the existence of gapless excitations in the CS phase.

\section{Dynamic structure factor and modular matrix}

In this section, we attempt to gain insight into the possible topological signature of the CS phase. We firstly calculate the dynamical structure factor (DSF) $\mathbb{S}(\mathbf{Q}, \omega)$, which encodes the information of the excitation spectrum that could be measured experimentally. It is defined via the spatiotemporal Fourier transform of the dynamical correlation:

$$
\begin{aligned}
\mathbb{S}(\mathbf{Q}, \omega)= & \int d t \sum_{i, j} \mathbf{S}_{i}(t) \cdot \mathbf{S}_{j}(0) \\
& \times \exp \left[-i \mathbf{Q} \cdot\left(\boldsymbol{R}_{i}-\boldsymbol{R}_{j}\right) t\right] \exp (-i \omega t) .
\end{aligned}
$$

The calculation is carried out on a 24-site hexagonal cluster by evaluating the continued fraction representation of the DSF. Methodologically, by preparing a special state to feed into the Lanczos algorithm, the tridiagonal matrix that is produced hides the coefficients of the continued fraction representation [63]. The energy resolution of $\omega$ during the DSF calculation is in steps of 0.001 and results in very sharp peaks for the finite system [64]. To plot the evolution of the DSF along the path $\boldsymbol{\Gamma}-\mathbf{X}-\boldsymbol{\Gamma}^{\prime}-\mathbf{X}^{\prime}-\boldsymbol{\Gamma}$ in the reciprocal space, we smeared in the energies $\omega$ by convolution of the results with a Gaussian distribution of width 0.1. The partial integrations of the DSF are also smeared by a Gaussian distribution but with a caveat, namely, the finite cluster has only specific allowed momenta $\mathbf{Q}$ where the DSF is calculated. After evaluating these allowed momenta, a Gaussian distribution is used to smear out the result for intermediate $\mathbf{Q}$ values.

We proceed in this way for the representative point at $\Gamma^{\prime}=0.15$ in the $\Gamma-\Gamma^{\prime}$ model. As can be seen from Fig. 9(a), there is a broad continuous feature in the low-frequency region reminiscent of a QSL despite the peak at the $\boldsymbol{\Gamma}^{\prime}$ point. This phenomenon is in contrast to the neighboring $\mathrm{AFM}_{c}$ phase whose DSF is discrete. Figures 9(b) and 9(c) show 



FIG. 9. The dynamical structure factor (DSF) at $\Gamma^{\prime} / \Gamma=0.15$ in the $\Gamma-\Gamma^{\prime}$ model. (a) Spectrum of the DSF $\mathbb{S}(\mathbf{Q}, \omega)$ at the highsymmetry points indicated in the left-bottom panel. (b) and (c) The integrated $\mathbb{S}(\mathbf{Q}, \omega)$ with respect to $\omega$ over the region of $[0.0,0.8]$ and $[0.15,0.35]$, respectively.

the integrated DSF intensities for two different energy windows of $\omega \in[0.0,0.8]$ and $\omega \in[0.15,0.35]$, respectively. The former is an analogy of the SSF where a peak at the $\Gamma^{\prime}$ point is spotted. For the latter case, the DSF is nonsymmetric under inversion, and the $C_{6}$ rotational symmetry is reduced to $C_{3}$ due to TRS breaking [65]. Generally, the TRS breaking could manifest itself in a $\mathbf{Q} \leftrightarrow-\mathbf{Q}$ asymmetry in the DSF

In the end, we checked for the topological order by calculating the modular $\mathcal{S}$ matrix in the CS phase [66]. Based on the central charge being 1 and a degenerate ground state of dimension 2, semion topological order is possible [67]. On each nontrivial cycle of the torus, we find the minimally entangled states own a twofold degeneracy. In all cases, we find the minimally entangled states on each cycle are nearly the same, leading to an identity matrix for the modular $\mathcal{S}$ matrix. This result is at odds with the assumption of a CS phase with topological order but is in line with a TRS-breaking CS ordering with short-range entanglement. However, we cannot make a definitive statement for this issue because of the small cluster used in our numerical calculations. Further details can be found in the SM [50].

\section{CONCLUSIONS}

In this paper, we have studied a $S=\frac{1}{2} J K \Gamma \Gamma^{\prime}$ model with dominant $\Gamma$ interaction on a honeycomb lattice, and in a wide proximate regime, we find a CS phase which owns a staggered scalar chirality on the two sublattices of the geometry and possesses a doubly degenerate ground state signifying TRS breaking. In this CS phase, its lowest energy gap has a tendency to close, consistent with entanglement entropy scalings on three-leg cylinders where a central charge $\sim 1$ is fitted. Further, the vanishing magnetization in a large enough system size indicates that the CS phase is a magnetically disordered state, in striking contrast to a classical CS ordering that possesses a long-range magnetic order. To unveil the nature of the CS phase, we constructed a minimally entangled state out of the degenerate doublet and calculated the modular $\mathcal{S}$ matrix, which turned out to be approximately an identity matrix. While we failed to probe the trail of the topological order on the small cluster, we cannot rule out the possibility that the CS phase is a gapless QSL, in which case the modular matrix is not well defined. This scenario is supported by the DSF calculation where a broad continuous feature in the lowfrequency region is observed. We hope that our discovery will stimulate further theoretical studies of the alluring CS phase.

\section{ACKNOWLEDGMENTS}

We thank S.-S. Gong and Y.-B. Kim for helpful discussion. Q.L. also appreciates X. Wang and J. Zhao for the collaboration on a related topic. This paper was supported by the NSERC Discovery Grant No. 06089-2016, the Centre for Quantum Materials at the University of Toronto, and the Canadian Institute for Advanced Research. H.-Y.K. also acknowledges funding from the Canada Research Chairs Program. Computations were performed on the GPC and Niagara supercomputers at the SciNet HPC Consortium. SciNet is funded by the Canada Foundation for Innovation under the auspices of Compute Canada, the Government of Ontario, Ontario Research Fund-Research Excellence, and the University of Toronto.
[1] W. Witczak-Krempa, G. Chen, Y. B. Kim, and L. Balents, Correlated quantum phenomena in the strong spin-orbit regime, Annu. Rev. Condens. Matter Phys. 5, 57 (2014).

[2] A. Banerjee, C. A. Bridges, J.-Q. Yan, A. A. Aczel, L. Li, M. B. Stone, G. E. Granroth, M. D. Lumsden, Y. Yiu, J. Knolle, S. Bhattacharjee, D. L. Kovrizhin, R. Moessner, D. A. Tennant, D. G. Mandrus, and S. E. Nagler, Proximate Kitaev quantum spin liquid behaviour in a honeycomb magnet, Nat. Mater. 15, 733 (2016).

[3] J. G. Rau, E. K.-H. Lee, and H.-Y. Kee, Spin-orbit physics giving rise to novel phases in correlated systems: Iridates and related materials, Annu. Rev. Condens. Matter Phys. 7, 195 (2016).
[4] H. Takagi, T. Takayama, G. Jackeli, G. Khaliullin, S. E. Nagler, Concept and realization of Kitaev quantum spin liquids, Nat. Rev. Phys. 1, 264 (2019).

[5] J. Wen, S.-L. Yu, S. Li, W. Yu, and J.-X. Li, Experimental identification of quantum spin liquids, npj Quantum Mater. 4, 12 (2019).

[6] D. Bergman, J. Alicea, E. Gull, S. Trebst, and L. Balents, Order by disorder and spiral spin liquid in frustrated diamond lattice antiferromagnets, Nat. Phys. 3, 487 (2007).

[7] Y. Shokef, A. Souslov, and T. C. Lubensky, Order by disorder in the antiferromagnetic Ising model on an elastic triangular lattice, Proc. Natl. Acad. Sci. USA 108, 11804 (2011).

[8] P. W. Anderson, Resonating valence bonds: A new kind of insulator? Mater. Res. Bull. 8, 153 (1973). 
[9] L. Balents, Spin liquids in frustrated magnets, Nature 464, 199 (2010).

[10] A. Kitaev, Anyons in an exactly solved model and beyond, Ann. Phys. (NY) 321, 2 (2006).

[11] J. G. Rau, E. K.-H. Lee, and H.-Y. Kee, Generic Spin Model for the Honeycomb Iridates beyond the Kitaev Limit, Phys. Rev. Lett. 112, 077204 (2014).

[12] V. M. Katukuri, S. Nishimoto, V. Yushankhai, A. Stoyanova, H. Kandpal, S. Choi, R. Coldea, I. Rousochatzakis, L. Hozoi, and J. van den Brink, Kitaev interactions between $j=\frac{1}{2}$ moments in honeycomb $\mathrm{Na}_{2} \mathrm{IrO}_{3}$ are large and ferromagnetic: Insights from $a b$ initio quantum chemistry calculations, New J. Phys. 16, 013056 (2014).

[13] G. Jackeli and G. Khaliullin, Mott Insulators in the Strong SpinOrbit Coupling Limit: From Heisenberg to a Quantum Compass and Kitaev Models, Phys. Rev. Lett. 102, 017205 (2009).

[14] F. Ye, S. Chi, H. Cao, B. C. Chakoumakos, J. A. FernandezBaca, R. Custelcean, T. F. Qi, O. B. Korneta, and G. Cao, Direct evidence of a zigzag spin-chain structure in the honeycomb lattice: a neutron and x-ray diffraction investigation of singlecrystal $\mathrm{Na}_{2} \mathrm{IrO}_{3}$, Phys. Rev. B 85, 180403(R) (2012).

[15] S. K. Choi, R. Coldea, A. N. Kolmogorov, T. Lancaster, I. I. Mazin, S. J. Blundell, P. G. Radaelli, Y. Singh, P. Gegenwart, K. R. Choi, S.-W. Cheong, P. J. Baker, C. Stock, and J. Taylor, Spin Waves and Revised Crystal Structure of Honeycomb Iridate $\mathrm{Na}_{2} \mathrm{IrO}_{3}$, Phys. Rev. Lett. 108, 127204 (2012).

[16] K. W. Plumb, J. P. Clancy, L. J. Sandilands, V. V. Shankar, Y. F. $\mathrm{Hu}, \mathrm{K} . \mathrm{S}$. Burch, H.-Y. Kee, and Y.-J. Kim, $\alpha-\mathrm{RuCl}_{3}$ : A spinorbit assisted Mott insulator on a honeycomb lattice, Phys. Rev. B 90, 041112(R) (2014).

[17] S. H. Chun, J.-W. Kim, J. Kim, H. Zheng, C. C. Stoumpos, C. D. Malliakas, J. F. Mitchell, K. Mehlawat, Y. Singh, Y. Choi, T. Gog, A. Al-Zein, M. M. Sala, M. Krisch, J. Chaloupka, G. Jackeli, G. Khaliullin, and B. J. Kim, Direct evidence for dominant bond-directional interactions in a honeycomb lattice iridate $\mathrm{Na}_{2} \mathrm{IrO}_{3}$, Nat. Phys. 11, 462 (2015).

[18] W. Wang, Z.-Y. Dong, S.-L. Yu, and J.-X. Li, Theoretical investigation of magnetic dynamics in $\alpha-\mathrm{RuCl}_{3}$, Phys. Rev. B 96, 115103 (2017).

[19] S. M. Winter, K. Riedl, P. A. Maksimov, A. L. Chernyshev, A. Honecker, and R. Valenti, Breakdown of magnons in a strongly spin-orbital coupled magnet, Nat. Commun. 8, 1152 (2018).

[20] I. Rousochatzakis and N. B. Perkins, Classical Spin Liquid Instability Driven by Off-Diagonal Exchange in Strong SpinOrbit Magnets, Phys. Rev. Lett. 118, 147204 (2017).

[21] P. Saha, Z. Fan, D. Zhang, and G.-W. Chern, Hidden Plaquette Order in a Classical Spin Liquid Stabilized by Strong OffDiagonal Exchange, Phys. Rev. Lett. 122, 257204 (2019).

[22] J. Wang, B. Normand, and Z.-X. Liu, One Proximate Kitaev Spin Liquid in the $K-J-\Gamma$ Model on the Honeycomb Lattice, Phys. Rev. Lett. 123, 197201 (2019).

[23] M. Gohlke, L. E. Chern, H.-Y. Kee, and Y. B. Kim, Emergence of nematic paramagnet via quantum order-by-disorder and pseudo-Goldstone modes in Kitaev magnets, Phys. Rev. Research 2, 043023 (2020).

[24] A. Catuneanu, Y. Yamaji, G. Wachtel, Y.-B. Kim, and H.-Y. Kee, Path to stable quantum spin liquids in spin-orbit coupled correlated materials, npj Quantum Mater. 3, 23 (2018).

[25] M. Gohlke, G. Wachtel, Y. Yamaji, F. Pollmann, and Y. B. Kim, Quantum spin liquid signatures in
Kitaev-like frustrated magnets, Phys. Rev. B 97, 075126 (2018).

[26] Q. Luo, J. Zhao, H.-Y. Kee, and X. Wang, Gapless quantum spin liquid in a honeycomb $\Gamma$ magnet, npj Quantum Mater. 6, 57 (2021).

[27] V. Kalmeyer and R. B. Laughlin, Equivalence of the Resonating-Valence-Bond and Fractional Quantum Hall States, Phys. Rev. Lett. 59, 2095 (1987).

[28] X. G. Wen, F. Wilczek, and A. Zee, Chiral spin states and superconductivity, Phys. Rev. B 39, 11413 (1989).

[29] H. Yao and S. A. Kivelson, Exact Chiral Spin Liquid with NonAbelian Anyons, Phys. Rev. Lett. 99, 247203 (2007).

[30] A. E. B. Nielsen, G. Sierra, and J. I. Cirac, Local models of fractional quantum Hall states in lattices and physical implementation, Nat. Commun. 4, 2864 (2013).

[31] B. Bauer, L. Cincio, B. P. Keller, M. Dolfi, G. Vidal, S. Trebst, and A. W. W. Ludwig, Chiral spin liquid and emergent anyons in a Kagome lattice Mott insulator, Nat. Commun. 5, 5137 (2014).

[32] S.-S. Gong, W. Zhu, and D. N. Sheng, Emergent chiral spin liquid: fractional quantum Hall effect in a kagome Heisenberg model, Sci. Rep. 4, 6317 (2014).

[33] Y.-C. He, D. N. Sheng, and Y. Chen, Chiral Spin Liquid in a Frustrated Anisotropic Kagome Heisenberg Model, Phys. Rev. Lett. 112, 137202 (2014).

[34] G. Ferraz, F. B. Ramos, R. Egger, and R. G. Pereira, Spin Chain Network Construction of Chiral Spin Liquids, Phys. Rev. Lett. 123, 137202 (2019).

[35] S.-S. Gong, W. Zheng, M. Lee, Y.-M. Lu, and D. N. Sheng, Chiral spin liquid with spinon Fermi surfaces in the spin- $\frac{1}{2}$ triangular Heisenberg model, Phys. Rev. B 100, 241111(R) (2019).

[36] A. Szasz, J. Motruk, M. P. Zaletel, and J. E. Moore, Chiral Spin Liquid Phase of the Triangular Lattice Hubbard Model: A Density Matrix Renormalization Group Study, Phys. Rev. X 10, 021042 (2020).

[37] J.-Y. Chen, S. Capponi, A. Wietek, M. Mambrini, N. Schuch, and D. Poilblanc, SU(3) 1 Chiral Spin Liquid on the Square Lattice: A View from Symmetric Projected Entangled Pair States, Phys. Rev. Lett. 125, 017201 (2020).

[38] Y.-F. Jiang and H.-C. Jiang, Topological Superconductivity in the Doped Chiral Spin Liquid on the Triangular Lattice, Phys. Rev. Lett. 125, 157002 (2020).

[39] R. Wang, Z. Y. Xie, B. Wang, and T. Sedrakyan, Theory of competing Chern-Simons orders and emergent phase transitions, arXiv:2101.04864.

[40] X.-G. Wen, Quantum Field Theory of Many-Body Systems: From the Origin of Sound to an Origin of Light and Electrons (Oxford University Press, Oxford, 2004).

[41] A. Ralko and J. Merino, Novel Chiral Quantum Spin Liquids in Kitaev Magnets, Phys. Rev. Lett. 124, 217203 (2020).

[42] A. Go, J. Jung, and E.-G. Moon, Vestiges of Topological Phase Transitions in Kitaev Quantum Spin Liquids, Phys. Rev. Lett. 122, 147203 (2019).

[43] Z.-X. Liu and B. Normand, Dirac and Chiral Quantum Spin Liquids on the Honeycomb Lattice in a Magnetic Field, Phys. Rev. Lett. 120, 187201 (2018).

[44] C. Hickey, L. Cincio, Z. Papić, and A. Paramekanti, Emergence of chiral spin liquids via quantum melting of noncoplanar magnetic orders, Phys. Rev. B 96, 115115 (2017). 
[45] Y. Huang, X.-Y. Dong, D. N. Sheng, and C. S. Ting, Quantum phase diagram and chiral spin liquid in the extended spin- $\frac{1}{2}$ honeycomb XY model, Phys. Rev. B 103, L041108 (2021).

[46] J. G. Rau and H.-Y. Kee, Trigonal distortion in the honeycomb iridates: proximity of zigzag and spiral phases in $\mathrm{Na}_{2} \mathrm{IrO}_{3}$, arXiv:1408.4811.

[47] S. R. White, Density Matrix Formulation for Quantum Renormalization Groups, Phys. Rev. Lett. 69, 2863 (1992).

[48] I. Peschel, X. Q. Wang, M. Kaulke, and K. Hallberg, DensityMatrix Renormalization (Springer, Berlin, 1999).

[49] E. M. Stoudenmire and S. R. White, Studying two dimensional systems with the density matrix renormalization group, Annu. Rev. Condens. Matter Phys. 3, 111 (2012).

[50] See Supplemental Material at http://link.aps.org/supplemental/ 10.1103/PhysRevResearch.4.013062 for details of the LUC orderings in the classical phase diagram of the $J K \Gamma \Gamma^{\prime}$ model, the scalar chirality, energy gap, and also the modular matrix of the CS phase.

[51] J. Chaloupka and G. Khaliullin, Hidden symmetries of the extended Kitaev-Heisenberg model: Implications for the honeycomb-lattice iridates $\mathrm{A}_{2} \mathrm{IrO}_{3}$, Phys. Rev. B 92, 024413 (2015).

[52] N. Metropolis, A. W. Rosenbluth, M. N. Rosenbluth, A. H. Teller, and E. Teller, Equation of state calculations by fast computing machines, J. Chem. Phys. 21, 1087 (1953).

[53] K. Hukushima and K. Nemoto, Exchange Monte Carlo method and application to spin glass simulations, J. Phys. Soc. Jpn. 65, 1604 (1996).

[54] H.-Y. Lee, R. Kaneko, L. E. Chern, T. Okubo, Y. Yamaji, N. Kawashima, and Y. B. Kim, Magnetic-field induced quantum phases in tensor network study of Kitaev magnets, Nat. Commun. 11, 1639 (2020).

[55] L. E. Chern, R. Kaneko, H.-Y. Lee, and Y. B. Kim, Magnetic field induced competing phases in spin-orbital entangled Kitaev magnets, Phys. Rev. Research 2, 013014 (2020).

[56] Z. Zhu, P. A. Maksimov, S. R. White, and A. L. Chernyshev, Disorder-Induced Mimicry of a Spin Liquid in $\mathrm{YbMgGaO}_{4}$, Phys. Rev. Lett. 119, 157201 (2017).

[57] J. S. Gordon, A. Catuneanu, E. S. Sørensen, and H.-Y. Kee, Theory of the field-revealed Kitaev spin liquid, Nat. Commun. 10, 2470 (2019).

[58] Q. Luo, J. Zhao, X. Wang, and H.-Y. Kee, Unveiling the phase diagram of a bond-alternating spin- $\frac{1}{2} K-\Gamma$ chain, Phys. Rev. B 103, 144423 (2021).

[59] P. A. Maksimov and A. L. Chernyshev, Rethinking $\alpha-\mathrm{RuCl}_{3}$, Phys. Rev. Research 2, 033011 (2020).

[60] E. C. Andrade, L. Janssen, and M. Vojta, Susceptibility anisotropy and its disorder evolution in models for Kitaev materials, Phys. Rev. B 102, 115160 (2020).

[61] P. Calabrese and J. Cardy, Entanglement entropy and quantum field theory, J. Stat. Mech. (2004) P06002.

[62] J. Eisert, M. Cramer, and M. B. Plenio, Colloquium: Area laws for the entanglement entropy, Rev. Mod. Phys. 82, 277 (2010).

[63] E. R. Gagliano and C. A. Balseiro, Dynamical Properties of Quantum Many-Body Systems at Zero Temperature, Phys. Rev. Lett. 59, 2999 (1987).

[64] P. Laurell and S. Okamoto, Dynamical and thermal magnetic properties of the Kitaev spin liquid candidate $\alpha-\mathrm{RuCl}_{3}$, npj Quantum Mater. 5, 2 (2020).

[65] J. C. Halimeh and M. Punk, Spin structure factors of chiral quantum spin liquids on the kagome lattice, Phys. Rev. B 94, 104413 (2016).

[66] Y. Zhang, T. Grover, A. Turner, M. Oshikawa, and A. Vishwanath, Quasiparticle statistics and braiding from groundstate entanglement, Phys. Rev. B 85, 235151 (2012).

[67] E. Rowell, R. Stong, and Z. Wang, On classification of modular tensor categories, Commun. Math. Phys. 292, 343 (2009). 